\title{
Total hip replacements following acetabular fractures. 7 to 15 years clinical and radiological results
}

\author{
Mohamed S. Kassem, Mostafa A. Elsayed
}

From the Elmansoura University Hospital, Elmansoura, Egypt and Elhadara University Hospital, Alexandria University, Egypt

This study is reporting the long term clinical and radiographic results of a group of 45 patients who underwent total hip arthroplasty following acetabular fractures. The study included 39 males and 6 females. The age of the patients ranged from 32 to 61 with a mean of 46.4 years. The indication for surgery was secondary osteoarthritis in 35 patients and avascular necrosis of the femoral head in the remaining ten. The follow up period ranged from 7 to 15 years with a median of 10.3 years. Uncemented total hip prostheses were used in 37 cases while 8 cases had hybrid prostheses with cemented cups and uncemented stems. Thirty patients $(66.7 \%)$ needed autogenous acetabular bone grafting. There has been a statistically significant improvement from a preoperative mean Oxford hip score of $\mathbf{1 6}$ to a postoperative mean score of $39.8(p<0.001)$. At the end of follow up, two cases had revision for cup loosening. The complications included one case of transient sciatic nerve palsy, and two cases of heterotopic ossification. Currently, total hip replacement remains the best option for end stage post traumatic arthritis. There are technical challenges associated with this replacement surgery which the surgeon should be aware of.

MeSH terms : arthroplasty; hip ; acetabulum.

Keywords : hip replacement ; acetabular fracture ; post traumatic arthritis.

\section{INTRODUCTION}

Acetabular fractures represent one of the major hip injuries which affect mostly the young active population. It usually results from road traffic accidents, but may result from other modes of injuries i.e. falling from height.

The complications which may occur following this fracture include secondary osteoarthritis of the hip joint as well as avascular necrosis of the head of the femur, which represent indications for total hip replacements. The old acetabular fracture indicated for hip arthroplasty could have been initially managed with either conservative treatment or surgical open reduction and internal fixation.

Matta and others have determined some factors which are expected to increase the possibility that any acetabular fracture will lead to hip replacement in the future including the severity of the causative force; the initial type of fracture; the age and weight of the patient; and the time lag from the

Mohamed S Kassem ${ }^{1}$,

Mostafa A Elsayed ${ }^{2}$

${ }^{1}$ Associate Professor of Orthopaedics, Alexandria University, Egypt. Consultant Orthopaedic Surgeon, Elhadara University Hospital, Alexandria, Egypt.

${ }^{2}$ Professor of Orthopaedics, Elmansoura University, Egypt. Consultant Orthopaedic Surgeon, Elmansoura University Hospital, Elmansoura, Egypt.

Correspondence : Mohamed Samir Kassem, Associate Professor of Orthopaedics, Orthopaedic department, Elhadara University Hospital, Elhadara, Lambrouzou, Alexandria, Egypt. PO Box: 21581. Phone : 00201001463603. Fax : 002034295792.

Email : mokassem21@gmail.com

o 2021, Acta Orthopædica Belgica. 
injury to the surgical procedure in case the initial management of the fracture was open reduction and internal fixation $(1,2)$.

Jimenez et al, in their review of literature, has concluded that long-term function of the hip joint may be compromised in many cases, regardless of the initial treatment choice (3).

Total hip replacements performed following acetabular fractures could entail surgical challenges starting from the exposure, going through the removal of any previous metalwork if necessary, reconstruction of the acetabular defects, and fixation of the prosthesis.

We are reporting our long term clinical and radiographic results of a group of 45 patients who underwent total hip arthroplasty following acetabular fractures including the difficulties and complications encountered.

\section{MATERIAL AND METHODS}

Forty five patients (39 males and 6 females) were included in this study. The age of the patients ranged from 32 to 61 with a mean of 41.4 years. The inclusion criteria were any patient presenting to our two hospitals with an old acetabular fracture complaining of pain which interferes with his daily activities to warrant hip replacement. The exclusion criteria included any patient with active infection related to the metal fixing his old acetabular fracture until this infection is cleared. The indication for surgery in 35 patients was secondary osteoarthritis while the indication for surgery in the remaining 10 patients was avascular necrosis of the femoral head. According to the type of acetabular fracture, isolated posterior wall fracture was the most common type present in 27 patients $(60 \%)$ (table I). According to the initial management, 36 patients had open reduction and internal fixation of their acetabular fractures while the remaining nine had conservative treatment. The time lag since the time of fracture till the replacement surgery ranged from 6 to 80 months with a mean of 33 months. Thirty four patients had shortening of the affected limb ranging from $0.5 \mathrm{~cm}$ up to $8 \mathrm{cms}$ with a mean of $3 \mathrm{cms}$.

The preoperative evaluation included plain radiographs, Computed tomography scan, as well as
Table I. - Distribution of the studied cases according to different studied parameters

\begin{tabular}{|c|l|}
\hline Age (years) & $41.40 \pm 7.11$ \\
\hline$\leq 40$ & $18(40.0 \%)$ \\
\hline$>40$ & $27(60.0 \%)$ \\
\hline Sex & \\
\hline Male & $39(86.7 \%)$ \\
\hline Female & $6(13.3 \%)$ \\
\hline Type of fracture & \\
\hline Post wall & $27(60.0 \%)$ \\
\hline Post wall post column & $6(13.3 \%)$ \\
\hline Post wall transverse & $3(6.7 \%)$ \\
\hline Post column & $6(13.3 \%)$ \\
\hline Both columns & $3(6.7 \%)$ \\
\hline Type of initial treatment & \\
\hline ORIF & $36(80.0 \%)$ \\
\hline Conservative & $9(20.0 \%)$ \\
\hline Indication & \\
\hline OA & $35(78.0 \%)$ \\
\hline AVN & $10(22.0 \%)$ \\
\hline Time lag & $33.0(6.0-80.0)$ \\
\hline
\end{tabular}

inflammatory markers to rule out infection in case of previous open reduction and internal fixation of the fracture.

Single stage surgery was performed in 39 patients while 6 patients had two stages surgery with removal of the implant as a first stage due to infection diagnosed either preoperatively (5 cases) with inflammatory markers or intraoperatively (one case) with infected tissue present around the metal. This was followed by prosthetic hip replacement as a second stage with a minimum interval of six weeks between the two stages and after the inflammatory markers ( $\mathrm{C}$ reactive protein, white cell count) became negative for infection for at least two consecutive weeks. All patients with previous open reduction and internal fixation of their acetabular fractures had intraoperative samples taken during their replacement surgery to rule out any occult infection.

The posterior Kocher Langenbeck approach was used in all cases. All or part of the metal work was removed at the replacement surgery in 10 cases. The type of the implant used was uncemented stem in all patients. One patient needed subtrochanteric femoral shortening of $2 \mathrm{cms}$ and a long stem uncemented stem to allow the hip to be reduced as it has been 
dislocated for 14 months. As regards the type of cup (cemented or uncemented) this was an intraoperative decision. The postoperative regimen included routine physiotherapy with gradual weight bearing depending on the use of bone graft to reconstruct any acetabular bone defects. Indomethacin was given to all patients postoperatively as a prophylaxis against heterotopic ossification.

The data collected and analysed included the operative time, the need to use bone graft, the length of hospital stay, the need for blood transfusion, and the complications. The patients were assessed clinically at the end of follow up period using Oxford Hip score. Radiographic assessment of the prostheses was also carried out at the end of follow up.

Data were fed to the computer and analyzed using IBM SPSS software package version 20.0. for normally distributed data, comparison between two studied groups were done using t-test while for more than two groups F-test (ANOVA) (LSD) was used, also paired t-test is used to analyze two paired data. Correlations between two quantitative variables were assessed using Pearson coefficients regarding normality of the data. Significance of the obtained results was judged at the $5 \%$ level.

\section{RESULTS}

The follow up period ranged from 7 to 15 years with a median of 10.3 years.

Uncemented total hip prostheses were used in 38 cases while 7 cases had hybrid prostheses with cemented acetabular cups and uncemented femoral stems (figure 1).

The operative time ranged from 100 minutes to 180 minutes with an average of 125 minutes. The mean operative time was more in the open reduction group than the conservative group (162 and 127 minutes respectively, $\mathrm{p}=0.011)$. Forty patients needed blood transfusion (89\%), and the average number of units was 2.5 units of red blood cells per case. The mean blood loss was more in the open reduction group than the conservative group (2153 and $1514 \mathrm{ml}$ respectively, $\mathrm{p}=0.014)$. The length of the hospital stay ranged from 4 to 8 days with a median of 5 days.
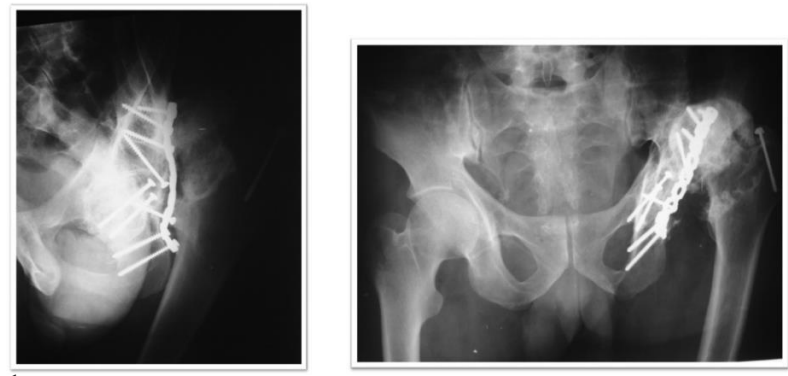

$1 \mathrm{a}$

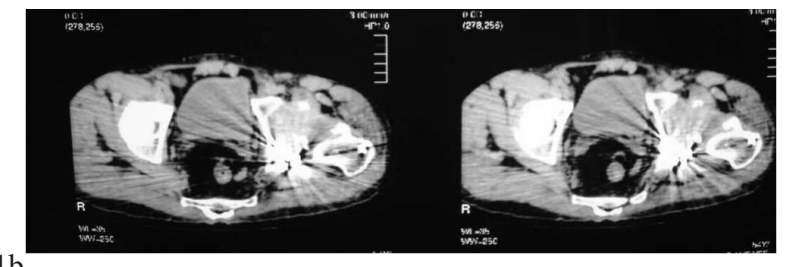

$1 \mathrm{~b}$

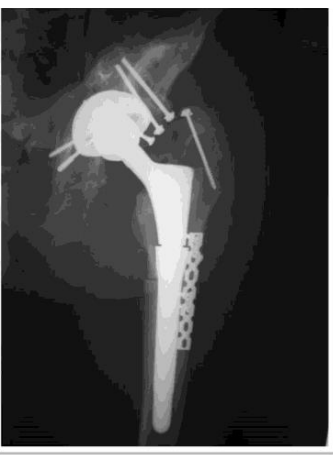

$1 \mathrm{c}$
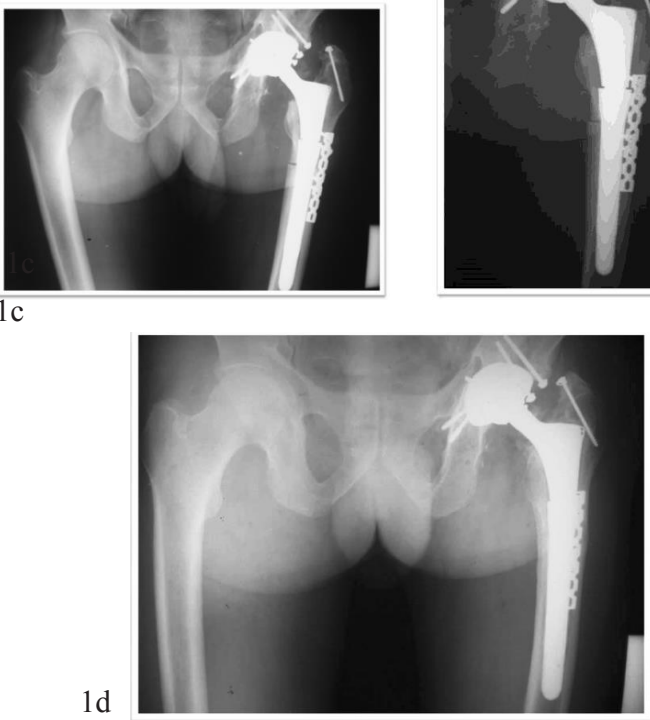

Fig. 1. - Plain radiographs showing malunited fracture acetabulum in 31 years old patient with secondary osteoarthritis a. preoperative radiograph; b. preoperative CT scan of the same patient ; c. immediate postoperative radiograph showing uncemented total hip replacement with subtrochanteric femoral shortening and uncemented modular stem and acetabular bulk autograft. ; d. 8 years follow up radiograph of the same patient

Thirty patients (66.7\%) needed autogenous acetabular grafting (18 patients impaction grafting and 12 patients bulk autograft as well as impaction grafting). 
Table II. - Comparison between preoperative and postoperative Oxford hip score

\begin{tabular}{|l|c|c|c|}
\hline Score & Preoperative & Postoperative & P \\
\cline { 1 - 3 } Min. - Max. & $8.0-24.0$ & $33.0-44.0$ & \multirow{2}{*}{$<0.001^{*}$} \\
\cline { 1 - 3 } Mean \pm SD & $16.0 \pm 4.12$ & $39.80 \pm 3.08$ & \\
\cline { 1 - 3 } Median & 16.0 & 41.0 & \\
\hline
\end{tabular}

As regards the Oxford hip score, there has been a statistically significant improvement from a preoperative mean score of 16 to a postoperative mean score of $39.8(\mathrm{p}<0.001)$ (table II).

There has been no significant correlation between the final outcome score and either the age, the sex, the type of acetabular fracture, the type of initial management, the indication for arthroplasty, the number of stages of the replacement surgery, the need for graft, and the type of replacement prosthesis. The relation between the preoperative and the postoperative Oxford score and the different studied parameters is listed in table III.

Radiographic assessment at the end of follow up showed good alignment of the components with good incorporation of the graft. There were 8 cases with residual shortening (five cases with $1 \mathrm{~cm}$ and the other 3 with $2 \mathrm{~cm}$ ) which was clinically not significant. One uncemented cup and one cemented cup became loose and were revised. Two cases had radioluscent line around an uncemented cup which was asymptomatic and without any radiological evidence of loosening.

The complications included one case of transient sciatic nerve palsy and two cases of heterotopic ossification. Two cases had revision of their acetabular cups (one cemented and the other uncemented) due to loosening 2 years and 3 years postoperatively respectively (figures 2 and 3 ).

Table III. — Relation between preoperative, postoperative score and different studied parameters

\begin{tabular}{|c|c|c|c|c|c|}
\hline & $\mathbf{N}$ & Preoperative score & $\mathrm{P}$ & Postoperative score & $\mathrm{p}$ \\
\hline \multicolumn{6}{|l|}{ Age (years) } \\
\hline$\leq 40$ & 18 & $17.50 \pm 6.19$ & 0.375 & $39.83 \pm 3.25$ & 0.974 \\
\hline$>40$ & 27 & $15.0 \pm 1.73$ & & $39.78 \pm 3.15$ & \\
\hline \multicolumn{6}{|l|}{ Sex } \\
\hline Male & 39 & $15.92 \pm 4.21$ & 0.862 & $39.62 \pm 3.04$ & 0.573 \\
\hline Female & 6 & $16.50 \pm 4.95$ & & $41.0 \pm 4.24$ & \\
\hline \multicolumn{6}{|l|}{ Type of fracture } \\
\hline Post wall & 27 & $16.56 \pm 3.0$ & $0.031^{*}$ & $39.67 \pm 3.84$ & 0.609 \\
\hline Post wall post column & 6 & $13.50 \pm 2.12$ & & $39.50 \pm 2.12$ & \\
\hline Post wall transverse & 3 & $8.0 \pm 0.0$ & & $40.0 \pm 0.0$ & \\
\hline Post column & 6 & $21.50 \pm 3.54$ & & $41.50 \pm 0.71$ & \\
\hline Both columns & 3 & $13.0 \pm 0.0$ & & $38.0 \pm 0.0$ & \\
\hline \multicolumn{6}{|c|}{ Type of intial management } \\
\hline ORIF & 36 & $16.33 \pm 4.14$ & 0.551 & $39.92 \pm 2.97$ & 0.955 \\
\hline Conservative & 9 & $14.67 \pm 4.62$ & & $39.33 \pm 4.16$ & \\
\hline \multicolumn{6}{|c|}{ Indication for arthroplasty } \\
\hline $\mathrm{OA}$ & 35 & $16.25 \pm 3.77$ & 0.656 & $39.33 \pm 3.17$ & 0.254 \\
\hline AVN & 10 & $15.0 \pm 6.24$ & & $41.67 \pm 2.08$ & \\
\hline \multicolumn{6}{|l|}{ Type of surgery } \\
\hline Single stage & 39 & $16.8 \pm 4.41$ & 0.862 & $39.31 \pm 2.98$ & 0.117 \\
\hline Two stages & 6 & $15.50 \pm 2.12$ & & $43.0 \pm 1.41$ & \\
\hline \multicolumn{6}{|l|}{ Type of graft } \\
\hline No & 15 & $17.0 \pm 4.0$ & 0.527 & $38.0 \pm 4.42$ & 0.250 \\
\hline Autogenous & 30 & $15.50 \pm 4.30$ & & $40.70 \pm 1.83$ & \\
\hline \multicolumn{6}{|l|}{ Type of prosthesis } \\
\hline Hybrid & 7 & $15.86 \pm 4.67$ & 0.906 & $39.57 \pm 2.94$ & 0.799 \\
\hline Uncemented & 38 & $16.13 \pm 3.91$ & & $40.0 \pm 3.38$ & \\
\hline
\end{tabular}




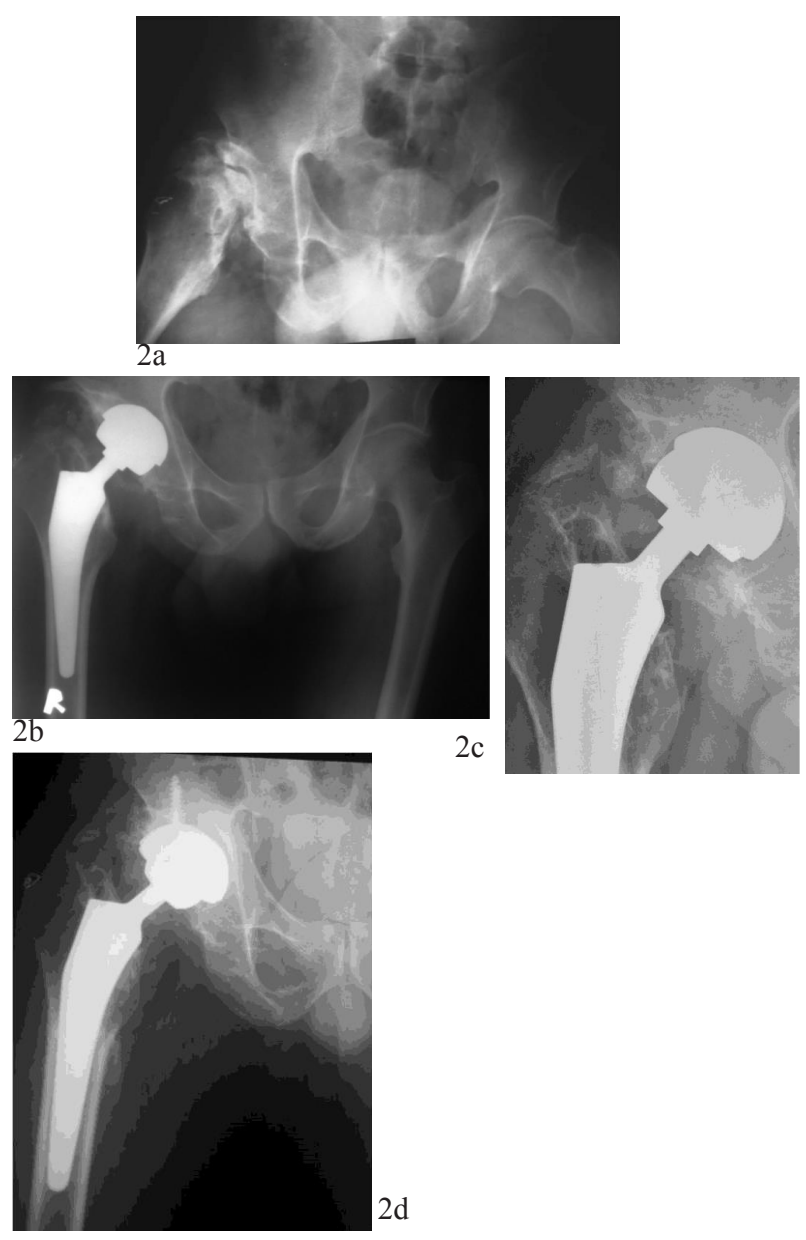

Fig. 2. - Plain radiographs of 46 years old patient with malunited fracture postoperative radiograph 4 years after revision of the cup showing Brooker grade III heterotropic ossification. a. acetabulum and secondary avascular necrosis of the hip ; b. preoperative radiograph showing Brooker grade I heterotropic ossification ; c. immediate postoperative radiograph; d. radiograph 3 years postoperative showing loose cup ; e. postoperative radiograph 4 years after revision of the cup showing Brooker grade III heterotropic ossification

Fig. 3. - Plain radiographs of 60 years old patient with united fracture acetabulum and secondary avascular necrosis of the hip. a. preoperative radiograph and CT Scan ; b. immediate postoperative radiograph showing hybrid total hip replacement with cemented cup and acetabular bulk autograft; c. radiograph 2 years postoperative showing loose cup ; d. postoperative radiograph 4 years after revision of the cup with allograft, acetabular cage, and cemented cup.

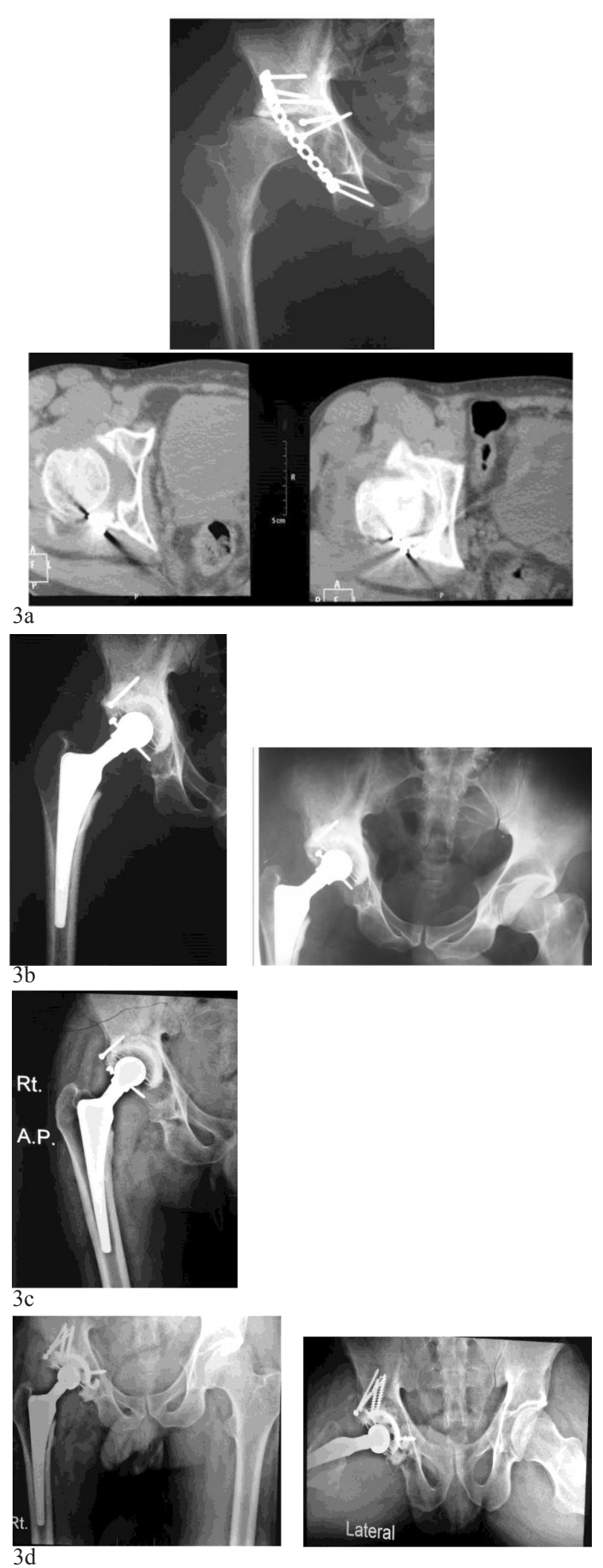

Acta Orthopædica Belgica, Vol. 87 - 3 - 2021 
There were no dislocations nor deep infections, nor thromboembolic events.

\section{DISCUSSION}

There has been a recent improvement in the management of acetabular fractures in the form of better understanding of its pathoanatomy, more accurate reduction through new approaches and techniques, as well as better fixation methods. However, despite this improvement, the incidence of post-traumatic osteoarthritis needing another procedure remains around $17 \%$ to $30 \%$ (4).

The arthrodesis option has become an unfavourable choice when counselled to the patients due to the restriction in movements leading to the arthroplasty option becoming the first choice in management of these arthritic hips (5).

Our mean follow up period of 10.3 years is considered a long term and is enough to give an idea about the clinical results, the patient satisfaction and to show any complications.

The average operative time of our cases was 125 minutes. This is considered longer than the average time of primary hip arthroplasty done for primary osteoarthritis (6). This increase in the operative time was related to the exposure especially in case of previous surgery caused by the scarring of the tissues, the heterotopic ossification, as well the need for removal of part of the metal work. This increase in the operative time was also related to the need for acetabular reconstruction in case of deficient acetabular bone stock.

As regards the need for blood transfusion, 40 patients needed perioperative transfusion which is also more than the need for transfusion in case of replacement for primary osteoarthritis (7). This increase in the blood loss could be explained by the scarring of the previous surgery and the longer operative time. Bellabarba et al have studied the results of 30 patients with total hip replacements for posttraumatic arthritis in 2001 and have reported that the operative time, the blood loss, and the perioperative transfusion requirements were greater in the patients with posttraumatic arthritis than they were in the patients with non-traumatic arthritis (8).
Our results showed that the operative time and the blood loss were more in the open reduction group than the conservative group. These results coincided with Bellabarba et al and Lai et al results $(8,9)$.

Thirty patients $(66.7 \%)$ needed autogenous bone graft to reconstruct the acetabulum. We used the preoperative CT scan to decide the need for impaction grafting or bulk autograft / allograft according to the type of the defects. We used the impaction grafting to fill the contained defects and the bulk autograft to reconstruct the uncontained defects. The bulk autograft was fixed with screws to the native ilium. We used uncemented acetabular shells with the bulk autograft along with or without shell screws fixing the shell to the native ilium and not to the graft to get primary cup fixation till late osteointegration occurs. Three patients initially treated conservatively needed impaction grafting while the other six patients needed both bulk autograft as well as impaction grafting. This could be explained by the fact that the restoration of the anatomy of the fractured acetabulum could be achieved more accurate with the open reduction management rather than the conservative management leading to the increased need for bone grafting in the conservative group in order to reconstruct the deficient acetabulum. In the case series of Bellabarba et al, two of the fifteen patients with a previous open reduction and internal fixation required bone-grafting of acetabular defects compared with seven of the fifteen patients treated by closed means (8). Romness et al reported in their review of 55 post acetabular fractures hip replacements, with 7 years follow up that there were no acetabular failures in the five hips in which acetabular bone grafting was performed either before or during the total hip arthroplasty (10). They concluded that the rates of component loosening and failure in patients undergoing total hip arthroplasty after an acetabular fracture are very much higher than the rates for routine arthroplasty for arthritis and attributed this increase to the damage or loss of acetabular bone stock in many of these patients. They suggested that reconstruction of the bony anatomy after fractures may have benefits with regard to subsequent arthroplasty even if it fails to prevent post-traumatic degeneration (10). 
This study included 38 cementless total hip replacements and 7 hybrid replacements with cemented cups and uncemented stems. The seven patients with cemented cups had intact acetabular walls which were considered intraoperatively thin and weak not to accommodate uncemented cups. The floor of the acetabulum was filled with impaction grafting after reaming of the acetabulum to remove any avascular bone and replacing it with autograft followed by good cementation technique with pressurisation of the cement into the acetabulum.

In our series, one cemented cup was revised due to loosening. This was attributed to the high position of the cup in relation to the centre of rotation of the hip as well as acetabular non-union which has led to loss of support and loosening of the cement mantle at two years. The cup was revised using allograft, acetabular ring, and a cemented cup. The other revised cup was an uncemented cup which failed three years post replacement due to loosening. The avascular acetabular bone floor may have been a contributing factor to the lack of osteointegration leading to the loss of fixation. We recommend the thorough debridement of the acetabular floor to remove all avascular bone, to graft the acetabular defects with autograft to increase the vascular bone stock, and to fix the uncemented cup with shell screws in case there is no adequate initial press fit.

There has a recent shift towards uncemented hip prostheses in replacing post acetabular fractures hips with secondary osteoarthritis $(9,11-14)$. We agree with many authors that hip replacements done acutely after acetabular fractures should be uncemented to allow the recent fracture to hea $(15,16)$. As regards the hip replacements done late for acetabular fractures, we think that there is still a place for cemented implants especially cups with proper cementation as long as there is no acetabular non-union. Moreover, we think that the current persistent relative high incidence of loosening following uncemented acetabular cups in posttraumatic arthritis is related to the use of uncemented cups in cases where satisfactory intraoperative primary stability could not be achieved making them more suitable for cemented cups with proper cementing technique $(16,17)$.
Two cases had radioluscent lines around their uncemented cup without any radiological evidence of loosening. The relatively early and increased incidence of acetabular radioluscent lines around acetabular cups have been reported in other case series $(11,12,16,17)$.

There has been residual shortening in 6 cases which was not clinically significant. The difficulty in restoring the normal leg length was related more to the open reduction group due to soft tissue scarring (5 cases).

The complications included one case of transient sciatic nerve palsy which recovered 12 months postsurgery. Mears et al have pointed out that the sciatic nerve injury could occur even with mild retraction in case the nerve was initially injured at the time of trauma or at the initial open reduction surgery (double crush syndrome) (2). This initial injury could have been occult without any significant clinical signs. We agree with their recommendation that surgical dissection around the nerve should be minimal during the replacement surgery and that removal of the previous metal work should be avoided if possible.

At the end of follow up, two patients suffered from Brooker grade III heterotopic ossification. One patient had open reduction and internal fixation of his initial acetabular fracture with Brooker grade II heterotopic ossification at the time of his replacement surgery. The other patient was initially managed conservatively with Brooker grade I at the time of surgery. They both did not have significant functional impairment at the time of last follow up. The current evidence support that indomethacin can prevent the formation of heterotopic ossification but no evidence supports it can stop the progress of heterotopic ossification once started (18).

There has been a statistically significant improvement in the Oxford Hip Score from a preoperative mean score of 16 to a postoperative mean score of 39.8. This mean postoperative score is considered less than the expected for primary hip arthroplasty (19-21). This could be explained by many factors including soft tissue scarring from previous surgery, poor acetabular bone stock, heterotopic ossification, and intrusive hardware. All the above mentioned factors make hip replacement 
after acetabular fractures a difficult and technically demanding surgery with results less than the replacement surgery done for primary osteoarthritis.

In their review of 68 hip replacements after acetabular fractures in 1978, Boardman and Charnley concluded that although the results were very good, the replacement surgery should be offered to this young population with caution (22). There has been a major improvement in the techniques of the arthroplasty since then especially related to the uncemented fixation and the design of the prostheses. Currently, total hip replacement remains the best option for end stage post traumatic arthritis. This type of replacement needs good preoperative clinical and radiological assessment. There are technical challenges associated with this replacement surgery which the surgeon should be aware of. Moreover, there is relative increased risk of some complications which the patient should be counselled about. The weaknesses of this study include being a retrospective study and the relative small number of patients.

\section{REFERENCES}

1. Matta, JM. Fractures of the acetabulum: accuracy of reduction and clinical results in patients managed operatively within three weeks after the injury. J. Bone and Joint Surg [Am] 1996 ; 78-A : 1632-1645.

2. Mears DC, Velyvis JH. Primary Total Hip Arthroplasty After Acetabular Fracture. J Bone Joint Surg [Am] 2000 ; 82 - A (9) : 1328-1328.

3. Jiminez ML, Tile M, Schenk RS. Total hip replacement after acetabular fracture. Orthop. Clin. North America 1997 ; $28: 435-446$.

4. Catalano JB, Born CT. Total hip arthroplasty after acetabular fracture treated initially with open reduction and internal fixation. Operative Techniques in Orthopaedics 1997 ; (7) : 250-255

5. Jauregui JJ, Kim JK, Shield WP 3rd, Harb M, Illical EM, Adib F, Maheshwari AV. Hip fusion takedown to a total hip arthroplasty-is it worth it? A systematic review. Int Orthop. 2017 Aug ; 41(8) :1535-1542.

6. Ross D, Erkocak O, Rasouli MR, Parvizi J. Operative Time Directly Correlates with Blood Loss and Need for Blood Transfusion in Total Joint Arthroplasty. Arch Bone Jt Surg. 2019 ; 7(3) :229-234.

7. Menezes, S., Manso, T., Seifert, I., Rodrigues, R., Gil, G. Blood loss in total hip/knee replacement surgery, European Journal of Anaesthesiology : June $2011 ; 28$ : 92
8. Bellabarba C, Berger RA, Bentley CD, et al. Cementless acetabular reconstruction after acetabular fracture. $J$ Bone Joint Surg [Am] 2001 ; 83-A(6) : 868-76.

9. Lai O, Yang J, Shen B, Zhou Z, Kang P, Pei F. Midterm Results of Uncemented Acetabular Reconstruction for Posttraumatic Arthritis Secondary to Acetabular Fracture. Journal of Arthroplasty 2011 ; (26) (7) : 1008-1013

10. Romness DW, Lewallen DG. Total hip arthroplasty after fracture of the acetabulum. Long-term results. J Bone Joint Surg Br. 1990 Sep ; 72(5) :761-4.

11. Huo MH, Solberg BD, Zatorski LE, Keggi KJ. Total hip replacements done without cement after acetabular fractures: A 4- to 8-year follow-up study. Journal of Arthroplasty 1999 ; (14) (7) : 827-831.

12. Ranawat A, Zelken J, Helfet D, Buly R. Total Hip Arthroplasty for Posttraumatic Arthritis after Acetabular Fracture. Journal of Arthroplasty 2009 ; (24) (5) : 759-767

13. Sermon A, Broos $P$, Vanderschot $P$. Total hip replacement for acetabular fractures: Results in 121 patients operated between 1983 and 2003. Injury 2008 ; (39) (8) : 914-921

14. Swanson MA, Knight JR, Huo MH. Total Hip Arthroplasty Following Previous Acetabular Fracture. Operative Techniques in Orthopaedics 2009 (19) (3) : 150154.

15. De Bellis UG, Legnani C, Maria Calori G. Acute total hip replacement for acetabular fractures : A systematic review of the literature. Injury 2014 ; (45) (2) : 356-361

16. Makridis KG, Obakponovwe O, Bobak $P$, Giannoudis PV. Total Hip Arthroplasty after Acetabular Fracture : Incidence of Complications, Reoperation Rates and Functional Outcomes : Evidence Today. Journal of Arthroplasty 2014 ; (29) (10) : 1983-1990

17. Zhang L,Zhou Y,Li Y, Xu H, Guo X, Zhou Y. Total Hip Arthroplasty for Failed Treatment of Acetabular Fractures : A 5-Year Follow-Up Study. Journal of Arthroplasty 2011 ; (26) (8) : 1189-1193

18. Baird EO, Kang QK. Prophylaxis of heterotopic ossification - an updated review. J Orthop Surg Res 2009 ; $20,4: 12$

19. Elsayed OM , Mohassab AM, Mazeed MG. Total Hip Replacement after Acetabular Fracture : A Review Article. The Egyptian Journal of Hospital Medicine. Oct 2017; 69 (3) : 2059-62

20. Refai HF, Kassem MS. The conventional anterolateral versus the minimally invasive direct anterior approached for total hip replacement. Panarab Journal of Orthopaedics and Trauma $2013 ; 17$ (2) : 75-82.

21. Refai HF, Kassem MS. The minimally invasive total hip replacement via the direct anterior approach : a short term clinical and radiological results. Alexandria Journal of Medicine, March 2014 (50) : 31-36.

22. Boardman KP, Charnley J. Low-friction arthroplasty after fracture-dislocations of the hip. J Bone Joint Surg Br 1978 ; $60: 495-7$. 